EPJ Web of Conferences 73, 04012 (2014)

DOI: $10.1051 /$ epjconf/20147304012

(C) Owned by the authors, published by EDP Sciences, 2014

\title{
Pion photo- and electroproduction in relativistic baryon ChPT
}

\author{
Lothar Tiator ${ }^{\mathrm{a}}$, Stefan Scherer and Marius Hilt \\ Institut für Kernphysik, Johannes Gutenberg-Universität Mainz, 55099 Mainz, Germany
}

\begin{abstract}
We present a calculation of pion photo- and electroproduction in manifestly Lorentz-invariant baryon chiral perturbation theory up to and including order $q^{4}$. We fix the low-energy constants by fitting experimental data in all available reaction channels. Our results can be accessed via a web interface, the so-called chiral MAID.
\end{abstract}

\section{Introduction}

In the middle of the 1980s, renewed interest in neutral pion photoproduction at threshold was triggered by experimental data from Saclay and Mainz, which indicated a serious disagreement with the predictions for the $s$-wave electric dipole amplitude $E_{0+}$ based on current algebra and PCAC. This discrepancy was explained with the aid of ChPT. Pion loops, which are beyond the currentalgebra framework, generate infrared singularities in the scattering amplitude which then modify the predicted low-energy expansion of $E_{0+}$. Subsequently, several experiments investigated pion photoand electroproduction in the threshold region were performed at Mainz, MIT-Bates, Saskatoon and TRIUMF, and on the theoretical side, all of the different reaction channels of pion photo- and electroproduction near threshold were extensively investigated by Bernard, Kaiser and Meißner within the framework of heavy-baryon chiral perturbation theory (HBChPT). For a complete list of references, see Ref. [1].

In the beginning, the manifestly Lorentz-invariant or relativistic formulation of ChPT (RChPT) was abandoned, as it seemingly had a problem with respect to power counting when loops containing internal nucleon lines come into play. Therefore, HBChPT became a standard tool for the analysis of pion photo- and electroproduction in the threshold region. In the meantime, the development of the infrared regularization (IR) scheme [2] and the extended on-mass-shell (EOMS) scheme [3] offered a solution to the power-counting problem, and RChPT became popular again.

We present a calculation of pion photo- and electroproduction on the nucleon in manifestly Lorentzinvariant baryon chiral perturbation theory up to and including chiral order $p^{4}$. Within this framework we analyze $\pi^{0}$ and charged photo- and electroproduction data in the threshold region, most of them obtained at the Mainz Microtron, MAMI. We also compare our results with the dynamical model DMT [4] and the unitary and causal effective field theory of Gasparyan and Lutz [5].

\footnotetext{
ae-mail: tiator@kph.uni-mainz.de
}

This is an Open Access article distributed under the terms of the Creative Commons Attribution License 4.0, which permits unrestricted use, distribution, and reproduction in any medium, provided the original work is properly cited. 

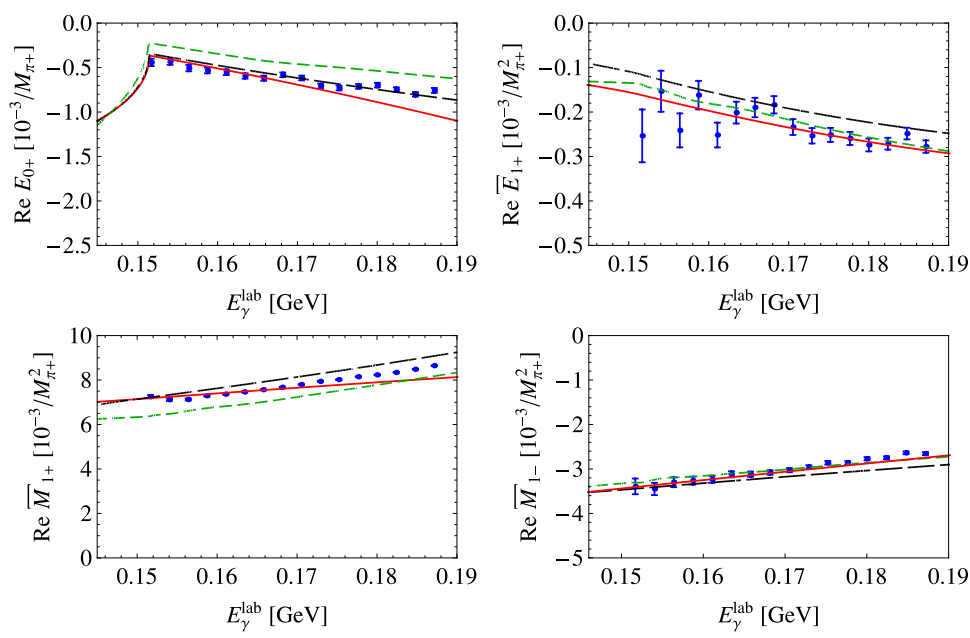

Figure 1. $S$ - and reduced $P$-wave multipoles for $\gamma+p \rightarrow p+\pi^{0}$. The solid (red) curves show our RChPT calculations at $O\left(q^{4}\right)$. The short-dashed (green) and long-dashed (black) curves are the predictions of the DMT model [4] and the GL model [5], respectively. The data are from Ref. [6].

\section{Pion photo- and electroproduction}

For pion photoproduction with polarized photons from an unpolarized target without recoil polarization detection, the cross section can be written in the following way with the unpolarized cross section $\sigma_{0}$ und the photon beam asymmetry $\Sigma$.

$$
\frac{d \sigma}{d \Omega}=\sigma_{0}\left(1-P_{T} \Sigma \cos 2 \varphi\right)
$$

For $\pi^{0}$ photoproduction on the proton, both observables are very precisely measured in the threshold region, allowing an almost model independent partial wave analysis [6].

For pion electroproduction, in the one-photon-exchange approximation, the differential cross section can be written as

$$
\frac{d \sigma}{d \mathcal{E}_{f} d \Omega_{f} d \Omega_{\pi}^{\mathrm{cm}}}=\Gamma \frac{d \sigma_{v}}{d \Omega_{\pi}^{\mathrm{cm}}},
$$

where $\Gamma$ is the virtual photon flux and $d \sigma_{v} / d \Omega_{\pi}^{\mathrm{cm}}$ is the pion production cross section for virtual photons.

For an unpolarized target and without recoil polarization detection, the virtual-photon differential cross section for pion production can be further decomposed as

$$
\frac{d \sigma_{v}}{d \Omega_{\pi}}=\frac{d \sigma_{T}}{d \Omega_{\pi}}+\epsilon \frac{d \sigma_{L}}{d \Omega_{\pi}}+\sqrt{2 \epsilon(1+\epsilon)} \frac{d \sigma_{L T}}{d \Omega_{\pi}} \cos \Phi_{\pi}+\epsilon \frac{d \sigma_{T T}}{d \Omega_{\pi}} \cos 2 \Phi_{\pi}+h \sqrt{2 \epsilon(1-\epsilon)} \frac{d \sigma_{L T^{\prime}}}{d \Omega_{\pi}} \sin \Phi_{\pi},
$$

where it is understood that the variables of the individual virtual-photon cross sections $d \sigma_{T} / d \Omega_{\pi}$ etc. refer to the $\mathrm{cm}$ frame. For further details, especially concerning polarization observables, see [7].

\section{Results and conclusions}

First, we show the real parts of the $S$ and $P$ waves in Fig. 1 together with single-energy fits of Ref. [6]. For comparison, we also show the predictions of the Dubna-Mainz-Taipei (DMT) model [4] and the covariant, unitary, chiral approach of Gasparyan and Lutz (GL) [5]. The multipole $E_{0+}$ agrees nicely 

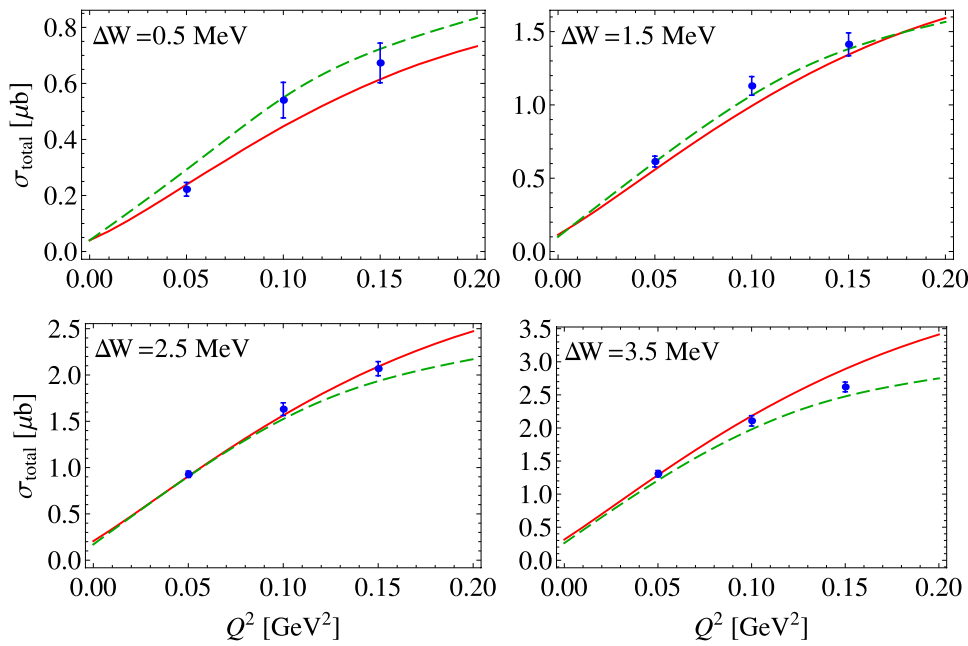

Figure 2. Total cross sections in $\mu \mathrm{b}$ as a function of $Q^{2}$ for different $\mathrm{cm}$ energies above threshold $\Delta W$ in $\mathrm{MeV}$. The data are from Ref. [8].

with the data in the fitted energy range. The reduced $P$ waves $\bar{E}_{1+}=E_{1+} / q_{\pi}$ and $\bar{M}_{1-}=M_{1-} / q_{\pi}$ with the pion momentum $q_{\pi}$ in the c.m. frame agree for even higher energies with the single energy fits. The largest deviation can be seen in $\bar{M}_{1+}$. This multipole is related to the $\Delta$ resonance and the rising of the data above $170 \mathrm{MeV}$ can be traced back to the influence of this resonance. As we did not include the $\Delta$ explicitly, this calculation is not able to fully describe its impact on the multipole.

For electroproduction, in Fig. 2 we show the total cross section $\sigma_{\text {total }}=\sigma_{T}+\epsilon \sigma_{L}$ in the threshold region together with the experimental data [8], and in Fig. 3 we compare our results for the coincidence cross sections $\sigma_{0}, \sigma_{T T}, \sigma_{L T}$ and the beam asymmetry $A_{L T^{\prime}}$ with the experimental data of Ref. [9] and the results of HBChPT [11] and the DMT model [4]. In general, the DMT model gives a very good description of all observables and amplitudes in the threshold region and can be used as a guideline for theoretical calculations in cases where experimental data do not exist. The HBChPT calculations shown in Fig. 3 were fitted to these data and are taken from Ref. [9]. In contrast, our RChPT calculation is not fitted to these data, as all LECs were already determined with other data before. While HBChPT gives a better description for the unpolarized cross section $\sigma_{0}\left(\Theta_{\pi}\right)=\sigma_{T}\left(\Theta_{\pi}\right)+\epsilon \sigma_{L}\left(\Theta_{\pi}\right)$ than our RChPT calculation, a comparison with the separated cross sections $\sigma_{T}$ and $\sigma_{L}$ shows that this is mainly due to a longitudinal cross section which is much too small in the HBChPT fit. For the other observables $\sigma_{L T}$, $\sigma_{T T}$, and the asymmetry $A_{L T^{\prime}}, \mathrm{RChPT}$ compares much better to the data than HBChPT. It is interesting to note that the asymmetry $A_{L T^{\prime}}$ depends only weakly on LECs and has an important contribution from the parameter-free pion loop contribution.

For $\Theta_{\pi}=\Phi_{\pi}=90^{\circ}$ and for $\epsilon \approx 1$ we find in very good approximation the simple form

$$
A_{L T^{\prime}}\left(90^{\circ}\right) \approx \frac{\sqrt{2 \epsilon(1-\epsilon)} \sqrt{Q^{2} / k_{0}^{2}}\left(-P_{2}\right) \operatorname{Im}\left(L_{0+}\right)}{P_{3}^{2}},
$$

where $P_{2}=3 E_{1+}-M_{1+}+M_{1-}$ and $P_{3}=2 M_{1+}+M_{1-}$. Therefore, this asymmetry is very sensitive to the imaginary part of the longitudinal $S$ wave $L_{0+}$, hence practically independent of LECs. This is very similar to the case of the target asymmetry $T$ for $\gamma p \rightarrow p \pi^{0}$ which we discussed in Ref. [10]. There, the target asymmetry is shown to be the ideal polarization observable to measure $\operatorname{Im}\left(E_{0+}\right)$.

In summary we have shown for the first time a chiral perturbation theory approach that consistently can describe all pion photo- and electroproduction processes in the threshold region equally well. Our 

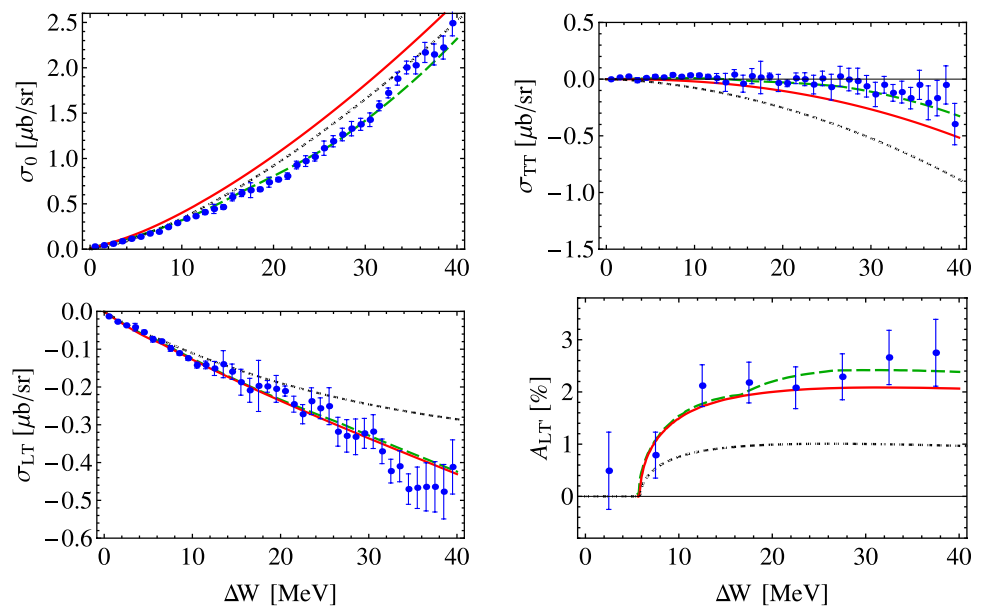

Figure 3. Coincidence cross sections $\sigma_{0}, \sigma_{T T}$, and $\sigma_{L T}$ in $\mu \mathrm{b} / \mathrm{sr}$ and beam asymmetry $A_{L T^{\prime}}$ in $\%$ at constant $Q^{2}=0.05 \mathrm{GeV}^{2}, \Theta_{\pi}=90^{\circ}, \Phi_{\pi}=90^{\circ}$, and $\epsilon=0.93$ as a function of $\Delta W$ above threshold. The solid (red) lines show our RChPT calculations at $O\left(q^{4}\right)$ and the dotted (black) lines are the heavy-baryon ChPT calculations of Ref. [11]. The dashed (green) curves are obtained from the DMT model [4]. The data are from Ref. [9].

relativistic chiral perturbation theory calculation is also online available within the MAID project as ChiralMaid under http://www.kph.uni-mainz.de/MAID/.

This work was supported by the Deutsche Forschungsgemeinschaft (SFB 443 and 1044).

\section{References}

[1] M. Hilt, B.C. Lehnhart, S. Scherer and L. Tiator, Phys. Rev. C 88, 055207 (2013)

[2] T. Becher and H. Leutwyler, Eur. Phys. J. C 9, 643 (1999)

[3] J. Gegelia and G. Japaridze, Phys. Rev. D 60, 114038 (1999)

[4] S.S. Kamalov, G.-Y. Chen, S.-N. Yang, D. Drechsel, and L. Tiator, Phys. Lett. B 522, 27 (2001)

[5] A. Gasparyan and M.F.M. Lutz, Nucl. Phys. A 848, 126 (2010)

[6] D. Hornidge et al. [A2 and CB-TAPS Collaboration], Phys. Rev. Lett. 111, 062004 (2013)

[7] D. Drechsel and L. Tiator, J. Phys. G 18, 449 (1992)

[8] H. Merkel, PoS CD 09, 112 (2009) and H. Merkel et al., [arXiv:1109.5075 [nucl-ex] ]

[9] M. Weis et al. [A1 Collaboration], Eur. Phys. J. A 38, 27 (2008)

[10] M. Hilt, S. Scherer and L. Tiator, Phys. Rev. C 87, 045204 (2013)

[11] V. Bernard, N. Kaiser, and U.-G. Meißner, Phys. Lett. B 383, 116 (1996) 